\title{
Ferramentas de digitalização 3D faça-você-mesmo na preservação do patrimônio cultural
}

\author{
Do-it-yourself 3D digitizing tools in preservation of cultural heritage \\ Outils de numérisation tridimensionnelle fais-le toi-même dans la \\ préservation du patrimoine culturel
}

\section{Herramientas de digitalización 3D hágalo-usted-mismo en la preservación del patrimonio cultural}

\author{
Yvana Oliveira Alencastro ${ }^{1}$ \\ Paulo Victor de Farias Dantas ${ }^{1}$ \\ Fábio Pinto da Silva ${ }^{1}$ \\ Jocelise Jacques de Jacques ${ }^{1}$
}

\author{
Recebido em 31/10/2017; revisado e aprovado em 02/02/2018; aceito em 13/02/2018 \\ DOI: http://dx.doi.org/10.20435/inter.v0i0.1744
}

\begin{abstract}
Resumo: Com o intuito de tornar a digitalização 3D mais acessível em museus, este estudo buscou por soluções apropriadas a um cenário de investimento reduzido. Baseando-se no faça-você-mesmo, a fotogrametria e a digitalização 3D por triangulação a laser foram testadas num ensaio com três objetos do Museu Júlio de Castilhos, Porto Alegre, Brasil. Ambas as técnicas mostraram bons resultados, a com base em fotogrametria, no entanto, apresentou mais vantagens, entre elas, uma menor curva de aprendizado.
\end{abstract}

Palavras-chave: digitalização 3D; faça-você-mesmo; patrimônio cultural; preservação.

Abstract: With the purpose of making 3D digitization more accessible in museums, this study looks into adequate solutions for a low-cost scenario. Based on a do-it-yourself perspective, photogrammetry and laser scanning techniques were experimented on three artefacts from the Júlio de Castilhos Museum, Porto Alegre, Brazil. Both techniques have shown great results, but the one based on photogrammetry presented more advantages, such as a smaller learning curve.

Keywords: 3D digitization; do-it-yourself; cultural heritage; preservation.

Résumé: Avec l'intention de rendre plus accessible la numérisation 3D dans les musées, cet étude a envisagé l'évaluation des techniques pertinentes sur un scénario d'investissement réduit. En se basant sur le modèle "fais-le toi-même", la photogrammétrie et la numérisation 3D par triangulation laser ont été testés dans un essai avec trois artefacts du Musée Júlio de Castilhos à Porto Alegre, Brésil. Après l'expérimentation, nous concluons que les deux techniques ont présentées des bons résultats. Par ailleurs la photogrammétrie a présenté plus d'avantage par rapport la numérisation 3D, à une plus petite courbe d'apprentissage.

Mots-clés: numérisation 3d; fais-le toi-même; patrimoine culturel; préservation.

Resumen: Con el fin de hacer la digitalización 3D en museos más accesible, este estudio buscó observar técnicas apropiadas a un escenario de inversión reducido. Baseándose em hágalo-usted-mismo, la fotogrametría y la digitalización 3D por triangulación láser fueron probadas en un ensayo con tres objetos en el Museo Júlio de Castilhos, Porto Alegre, Brasil. Después del experimento, se concluyó que ambas técnicas mostraron buenos resultados, sin embargo la fotogrametría presenta más ventajas, entre ellas, la menor curva de aprendizaje.

Palabras clave: digitalización 3D; hágalo-usted-mismo; patrimonio cultural; preservación.

\footnotetext{
${ }^{1}$ Universidade Federal do Rio Grande do Sul (UFRGS), Porto Alegre, Rio Grande do Sul, Brasil.
} 


\section{INTRODUÇÃO}

A digitalização tridimensional (3D) vem se tornando importante ferramenta em projetos de restauração e difusão do patrimônio cultural. Como, por exemplo, o The Digital Michelangelo Project, no qual diversas esculturas de Michelangelo foram digitalizadas tridimensionalmente, incluindo a famosa estátua David (LEVOY et al., 2000). Outro exemplo, é o projeto Aleijadinho Digital, realizado em Congonhas dos Campos, MG, que digitalizou 30 obras desse artista mineiro (ANDRADE et al., 2012; GOMES, 2016). Em Porto Alegre, RS, o monumento símbolo da cidade, "O Laçador", foi digitalizado tridimensionalmente para fins de preservação e pesquisa (SILVA; KINDLEIN JUNIOR, 2013). Já o Museu Nacional, no Rio de Janeiro, tem feito uso da digitalização 3D, modelagem 3D e fabricação digital como forma de modernizar processos, incluindo a interação com o público visitante (AZEVEDO et al., 2011).

Instituições voltadas ao patrimônio cultural podem se beneficiar de modelos digitais 3D no estudo de objetos (históricos/arqueológicos), na documentação e catalogação, na preservação, e na restauração (GUARNIERI; PIROTTI; VETTORE, 2010). Segundo Muchacho (2009), objetos digitalizados possibilitam novas formas de ver e interpretar acervos, auxiliando também em pesquisas, na comunicação e na divulgação.

Devido ao custo de projetos de digitalização 3D, o objetivo deste artigo foi verificar as possibilidades de utilização de ferramentas de digitalização 3D faça-você-mesmo ${ }^{2}$ para fins de preservação do patrimônio cultural em museus. Essas ferramentas, além de reduzir o custo de implementação e manutenção, tendem a ser de fácil aprendizado, e podem ser utilizadas dentro da reserva técnica do museu, adaptando-se às necessidades, ao tipo de acervo e ao espaço físico, e podem ser executadas pelo corpo técnico que detém o conhecimento das fragilidades características de cada peça. Desse modo, acredita-se que essas ferramentas podem favorecer a documentação do patrimônio, possibilitando a reprodução do acervo em meio virtual e a conservação das coleções, evitando também o deslocamento para laboratórios especializados e eventuais acidentes.

Para fins desta pesquisa, foram digitalizados três objetos do Museu Júlio de Castilhos, em Porto Alegre, RS, com dois sistemas de digitalização 3D faça-você-mesmo: um baseado em triangulação a laser, e outro, em fotogrametria. Objetivou-se verificar se essas técnicas atendem às demandas de preservação do patrimônio cultural em museus.

\subsection{Digitalização 3D}

Digitalização 3D é o nome dado a um conjunto de técnicas pelas quais é possível capturar a forma geométrica de um objeto físico, de modo a reproduzi-la digitalmente. Durante o processo, coordenadas $(x, y z)$ de pontos sobre a superfície do objeto são medidas para se obter, geralmente, uma nuvem de pontos que descreve sua forma tridimensional. Além da forma, ainda é possível capturar outras características do objeto como a cor aparente e o comportamento de refletância ${ }^{3}$, que podem ser projetadas sobre a geometria obtida (BERNARDINI; RUSHMEIER, 2002; EBRAHIM, 2011).

\footnotetext{
${ }^{2}$ Acrônimo do inglês DIY (do-it-yourself), relativo a atitude anticonsumista na qual o indivíduo, em vez de pagar por um produto, busca alternativas de produzi-lo por conta própria (LEMOS, 2004).

${ }^{3}$ Relação entre o fluxo de radiação que incide numa superfície e o que é refletido (REFLETÂNCIA, s.d.).
} 
De acordo com Pavlidis et al. (2007), a digitalização 3D é um processo composto por três fases principais: (a) preparação - decisões como a escolha da técnica e metodologia a ser adotada, o local da digitalização, planejamento de segurança; (b) aquisição digital - quando ocorre a captura de dados da superfície do objeto através de varreduras; (c) processamento de dados - modelagem do objeto através de processos como unificação de varreduras, processamento de dados geométricos e processamento de dados de cor.

A escolha da técnica depende da finalidade do modelo digitalizado, do orçamento disponível, do tamanho, forma, nível de detalhe e matérias-primas do objeto (PAVLIDIS et al., 2007). Entre as técnicas de digitalização 3D faça-você-mesmo, destacam-se ferramentas para digitalização por triangulação a laser e fotogrametria. Estas apresentam uma variedade de softwares e modelos de hardware disponibilizados gratuitamente ou a baixo custo (REPRAP, 2016). As duas técnicas estão entre as indicadas por Pavlidis et al. (2007) para o uso na digitalização 3D de objetos de patrimônio cultural.

\subsubsection{Digitalização 3D por triangulação a laser}

O procedimento de digitalização 3D por triangulação a laser consiste em um sistema com um emissor laser e um detector óptico (geralmente uma câmera digital). O emissor laser projeta uma linha na superfície do objeto e o detector registra a variação dessa linha durante a varredura. A partir dessa variação, o software calcula as coordenadas de pontos por meio de triangulação, ou seja, do triângulo formado entre emissor, detector e objeto. Para a reconstrução completa do objeto tridimensional, dependendo da complexidade, podem ser necessárias múltiplas varreduras (BERNARDINI; RUSHMEIER, 2002; PAVLIDIS et al., 2007; EBRAHIM, 2011; WEINMANN; KLEIN, 2015).

Pavlidis et al. (2007) apontam como vantagem da digitalização a laser a sua alta precisão geométrica. Alguns sistemas a laser, no entanto, não extraem informações de cor, e não apresentam resultados aceitáveis para determinadas superfícies materiais, como as com elevado grau de refletância especular ou transmitância ${ }^{4}$. Sistemas capaz de lidar com uma maior gama de superfícies materiais apresentam custos mais elevados.

\subsubsection{Fotogrametria}

Na digitalização por fotogrametria, a captura de dados geométricos é realizada a partir de uma sequência de imagens fotográficas, obtidas de diferentes pontos de vista. Durante o processamento, o software realiza o alinhamento tridimensional das imagens capturadas com base na detecção de sobreposições e pontos equivalentes, determinando, dessa maneira, a posição e orientação da câmera relativa ao objeto fotografado. A partir dessa orientação é realizada a triangulação de diversos pontos detectados, restituindo assim a geometria tridimensional do objeto, em forma de nuvem de pontos ou malha tridimensional (TOMMASELLI et al., 1999; SCHENK, 2005; YASTIKLI, 2007).

Salienta-se que a configuração correta da câmera, com respeito às características do objeto (tamanho, detalhes, cores), é de extrema importância. Conforme Dantas et al. (2016), o resultado a ser obtido com essa técnica depende da qualidade das imagens adquiridas. Percoco,

\footnotetext{
${ }^{4}$ Razão entre a quantidade de luz que atravessa um material e a que sobre ele incide (BRASIL, 2006).
} 
Lavecchia e Salmerón (2015) reiteram a importância do foco e que, para objetos pequenos, é necessária uma lente macro.

Como vantagem, Pavlidis et al. (2007) afirmam que a fotogrametria pode ser utilizada até em objetos complexos com alto nível de detalhamento na superfície. Todavia é necessário um ambiente com iluminação controlada para aquisição das fotografias.

\section{PROCEDIMENTOS METODOLÓGICOS}

Para verificar se a digitalização 3D faça-você-mesmo pode ser utilizada para fins de preservação do patrimônio cultural em museus, ensaiou-se o uso da digitalização 3D por triangulação a laser e por fotogrametria dentro da reserva técnica do Museu Júlio de Castilhos, Porto Alegre, RS.

Devido à natureza experimental dos métodos faça-você-mesmo empregados, foram tomadas como referência as normas de conservação preventiva sobre a incidência luminosa para a seleção das peças a serem digitalizadas. Essas normas estabelecem um limite de iluminância e radiação UV do ambiente no qual as peças permanecem para conservação, de acordo com os materiais que as compõem (SOUZA; FRONER, 2008). Desse modo, foram selecionadas três peças de pequeno porte para os experimentos, sendo duas cerâmicas e uma metálica que não apresentam sensibilidade à luz segundo as normas (figura 1). Essas peças foram pré-selecionadas através do sistema de documentação Donato ${ }^{5}$, do museu.

Figura 1 - Peças selecionadas

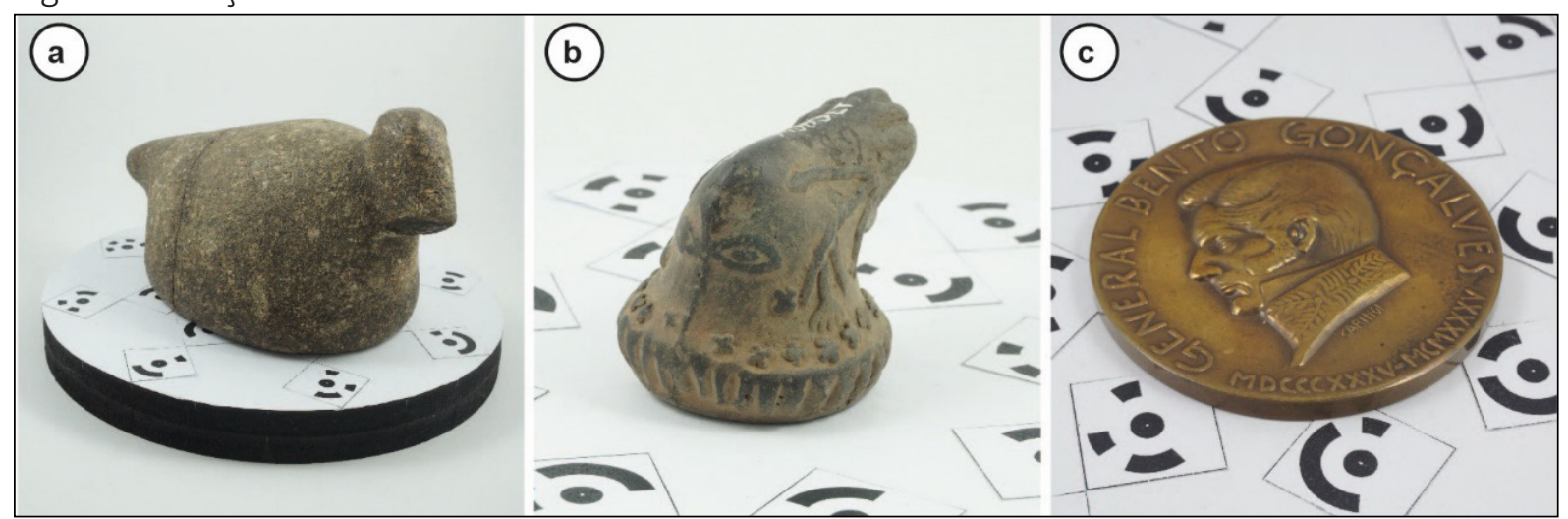

a) Zoólito pré-histórico

b) Cachimbo antropomórfico

c) Medalha comemorativa

Fonte: Elaborada pelos autores.

a) Zoólito pré-histórico (1 DC.), encontrado na cidade de Osório-RS, em pedra polida (170x95x72mm).

b) Cachimbo antropomorfo, origem e datas desconhecidas, em cerâmica ( $45 \times 41 \times 33 \mathrm{~mm}$ ).

c) Medalha comemorativa do centenário Farroupilha, 1935, cunhada em metal (65×65×3mm).

Para observar se as técnicas de digitalização 3D faça-você-mesmo selecionadas podem ser utilizadas para fins de preservação do patrimônio cultural, foram utilizadas como base os critérios estabelecidos por Pavlidis et al. (2007): custo, material do objeto, tamanho do objeto, portabilidade, precisão do sistema, aquisição de textura, produtividade e habilidade do usuário.

${ }^{5}$ O Sistema Donato/SIMBA foi desenvolvido para gerenciar informações do acervo do Museu Nacional de Belas Artes e foi liberado para diversos museus brasileiros. 
Ao final, reproduziram-se os modelos numa impressora 3D Cliever CL1, com tecnologia de fusão e deposição de material ${ }^{6}$ (FDM), verificando a possibilidade de reprodução.

\subsection{Estrutura para digitalização 3D por triangulação a laser}

Dentre os softwares de digitalização por triangulação a laser faça-você-mesmo (REPRAP, 2016), o David Laserscanner 3D apresenta versões de avaliação e comercial. O modo de avaliação é gratuito, nele é possível realizar todo o processo de digitalização, no entanto não é possível salvar o projeto, e a exportação dos modelos é limitada à baixa resolução. Ele pode funcionar de forma automatizada, porém a configuração que requer menor investimento é a de varredura manual, enquanto a superfície é capturada por uma webcam e identificada pelo algoritmo de triangulação (SILVA, 2011), como pode ser visualizado na figura 2.

Figura 2 - Montagem da técnica

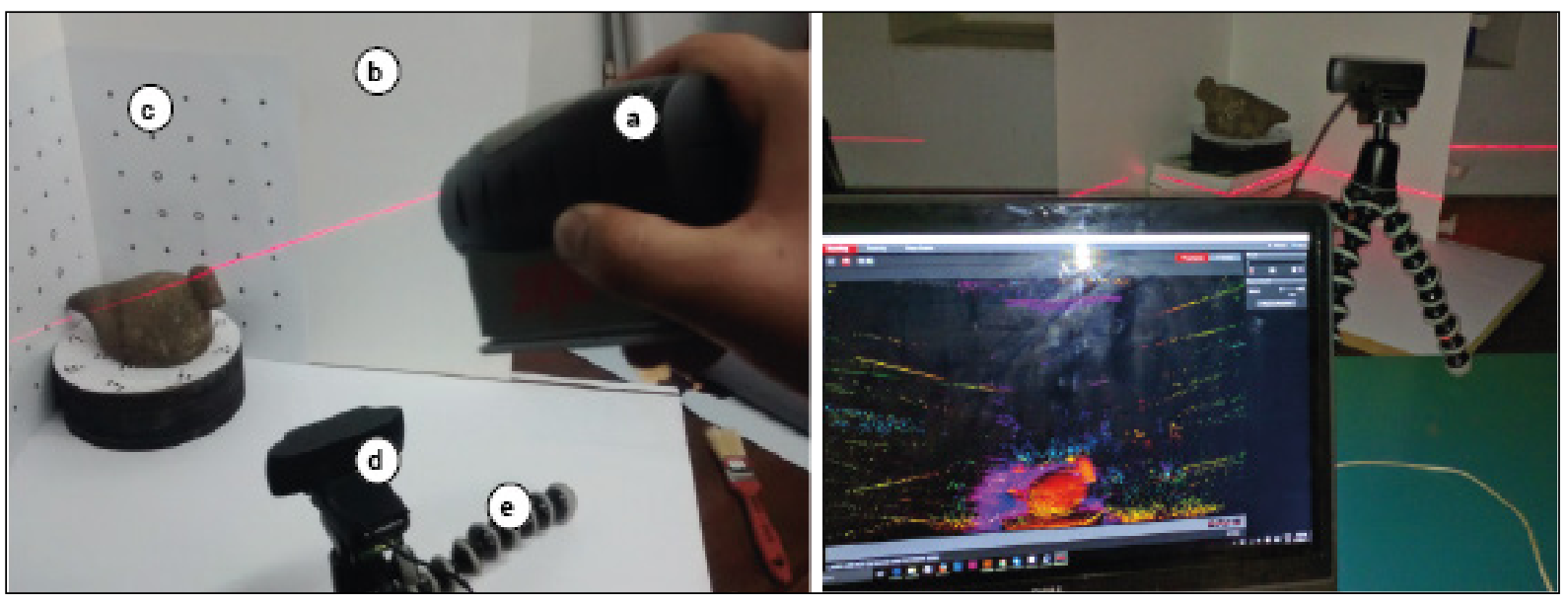

a) laser; b) painéis; c) marcas de calibragem; d) webcam; e) tripé

Fonte: Elaborada pelos autores.

O ambiente foi preparado com a utilização do programa David Laserscanner 3D, conforme a figura 2. Foram utilizados (a) um laser de mão, (b) dois painéis de MDF perpendiculares, (c) marcas de calibração disponibilizadas pelo software, (d) webcam, (e) tripé e um computador para receber a gravação da câmera. Os modelos dos equipamentos utilizados foram: webcam Logitech ${ }^{\circledR}$ C920 HD (1920 x 1080 pixels, widescreen 16:9 e 30 quadros por segundo); nivelador laser Skill ${ }^{\circledR} 500$ (laser linear ${ }^{7}$ vermelho, comprimento de onda $650 \mathrm{~nm}, 100$ lux).

O David 3D precisa ser calibrado antes da realização da varredura. As marcas de calibração devem compor uma área maior que o objeto que será digitalizado. A webcam precisa enquadrar essas marcas deixando uma pequena moldura. Durante a calibragem, a luz ambiente deve permanecer acesa para melhor identificação das marcas de calibração.

Para iniciar a digitalização, recomenda-se a redução da luz ambiente, de modo a aumentar o contraste do feixe do laser. A exposição da câmera também deve ser ajustada com o mesmo propósito. O objeto deve ser colocado dentro da área triangular formada pelos dois painéis e o

\footnotetext{
${ }^{6}$ Fabricação aditiva através de extrusão termoplástica camada-a-camada (BOSCHETTO; BOTTINI; VENIALI, 2016).

${ }^{7}$ Laser cuja reflexão tem forma de linha.
} 
fim da marca de calibração. O laser deve incidir igualmente sobre os painéis para ser reconhecido pelo software. Conforme o laser incide no objeto, o David 3D realiza o registro dos pontos da superfície e apresenta o modelo 3D sendo gerado.

Para gerar o modelo 3D, é preciso realizar varreduras em diferentes posições do objeto. Em seguida, o software alinha a nuvem de pontos das diferentes varreduras, oferecendo a possibilidade de ajuste manual, e salvamento do projeto para a conclusão posterior e melhorias.

\subsection{Estrutura para digitalização por fotogrametria}

Para o experimento da fotogrametria com o uso do programa 123D Catch, o ambiente foi preparado de acordo com a Figura 3. Foi utilizada (a) uma câmera Sony A6000 (resolução máxima de 6000 × 4000 pixels) com (b) filtro polarizador circular, (c) tripé de mesa, (d) um miniestúdio com fundo infinito faça-você-mesmo de 400 x 600 mm, (e) difusores de luz, (f) duas luminárias de mesa com lâmpadas fluorescentes brancas de 15 W, 80 lux (somadas antes de passar pelo difusor) e (g) base circular com marcadores para auxiliar no alinhamento do software e também no giro dos objetos.

Durante a captura de imagens, é realizada uma sequência de aquisição com variação angular constante, até completar 360 em torno do objeto. Dependendo da complexidade do objeto, pode ser necessário realizar diversas sequências em posições diferentes, até obter uma cobertura completa de sua superfície.

Em seguida, as imagens são processadas em nuvem com o uso do software 123D Catch, em um computador com acesso à internet, onde são convertidas em modelos 3D. Por fim, é necessário o download e um pós-processamento com o uso de software de modelagem para a conclusão do objeto digitalizado. O software 123D Catch está dentre os programas que dão suporte à técnica de fotogrametria faça-você-mesmo (REPRAP, 2016) e destaca-se por ter licença gratuita.

Figura 3 - Montagem da técnica

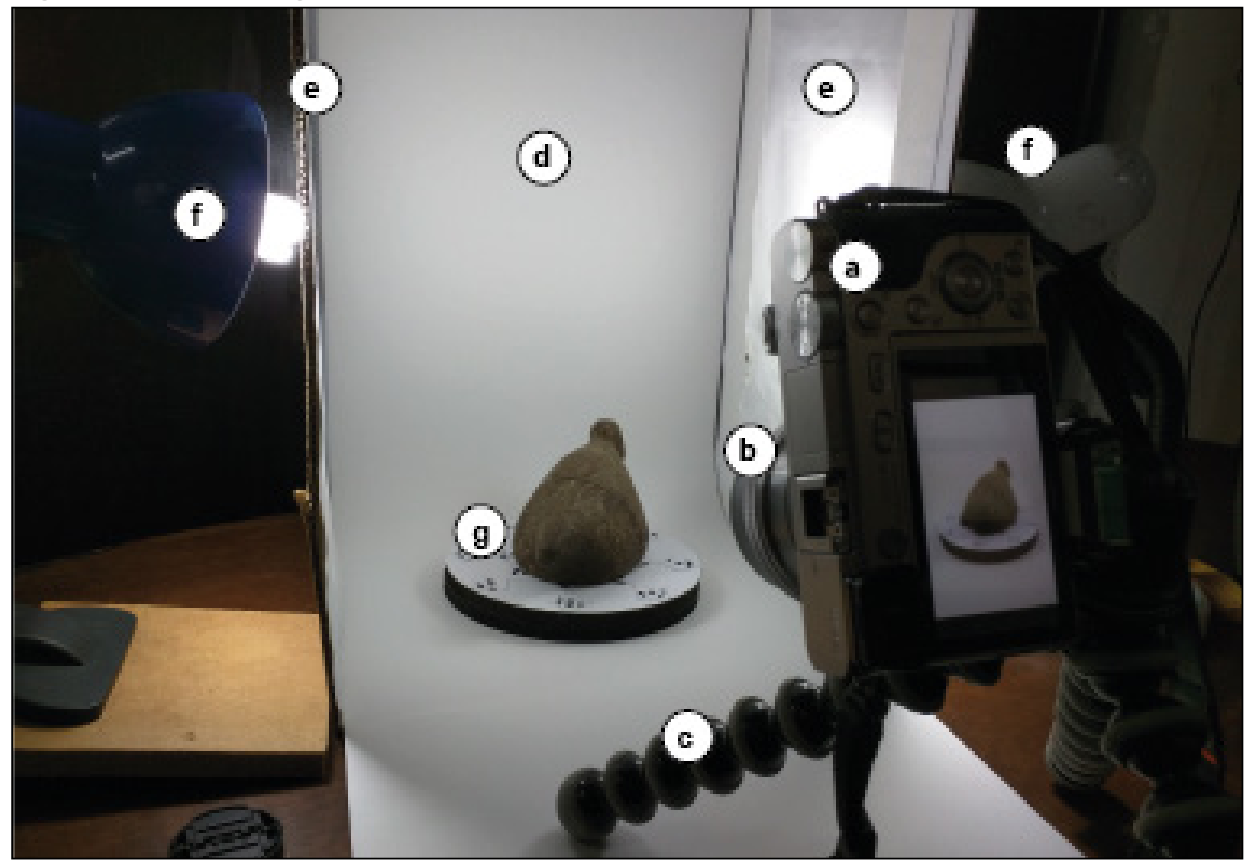

a) câmera fotográfica; b) filtro polarizador; c) tripé; d) estúdio; e) difusores; f) luminárias; g) base.

Fonte: Elaborada pelos autores. 


\section{RESULTADOS}

Ambas as técnicas atenderam aos critérios de Pavlidis et al. (2007) ao considerar uma perspectiva que necessite de menor precisão geométrica ${ }^{8}$.

\subsection{Digitalização com a utilização do David laserscanner 3D}

Para a realização da digitalização a laser, realizaram-se quatro tentativas para obter modelos representativos. Na primeira tentativa de uso do laser, foram realizadas dez varreduras em diferentes posições, todas geraram arquivos com pouco volume de informação. Foi verificado que as marcas de calibragem, 515 × 325 mm, estavam grandes para o zoólito; desse modo, a webcam precisou ficar mais distante reduzindo a resolução da captura.

Na segunda tentativa, foram utilizadas novas marcas de calibragem com $270 \times 170 \mathrm{~mm}$. Nenhuma varredura pôde ser concluída, pois, ao reduzir as marcas de calibragem e aproximar a câmera, foi reduzida também a distância focal e, consequentemente, a profundidade de campo. Assim, o Zoólito não ficou dentro de uma área de foco suficiente para captar informações (figura 4a).

Para a terceira tentativa, foi utilizada a mesma base de calibragem do ensaio anterior, porém foi realizada com o cachimbo, um objeto menor (figura 4b). O software realizou a leitura completa. Foram geradas 14 varreduras possibilitando alinhamento e fusão do modelo no próprio software.

A quarta tentativa foi realizada com a medalha e o mesmo calibrador (figura 4c). Foram realizadas três varreduras e todas as três apresentaram uma quantidade grande de falhas na superfície, mesmo utilizando um pó revelador branco para deixar a superfície do metal mais opaca. Ao final dos testes, verificou-se que a calibração havia perdido precisão devido a uma instabilidade nos painéis.

O software David laserscanner 3D realiza a varredura, monta a nuvem de pontos e capta as cores reduzindo o processamento em outro software. Porém, na versão de avaliação, a resolução final é reduzida, e a textura não é salva. O tempo total do processo foi de aproximadamente 16 horas.

\footnotetext{
${ }^{8}$ Exemplo: exige-se alta precisão geométrica em projetos nos quais uma mínima variação no formato do objeto resultaria no mal funcionamento de um maquinário.
} 
Figura 4 - Objetos digitalizados e impressos
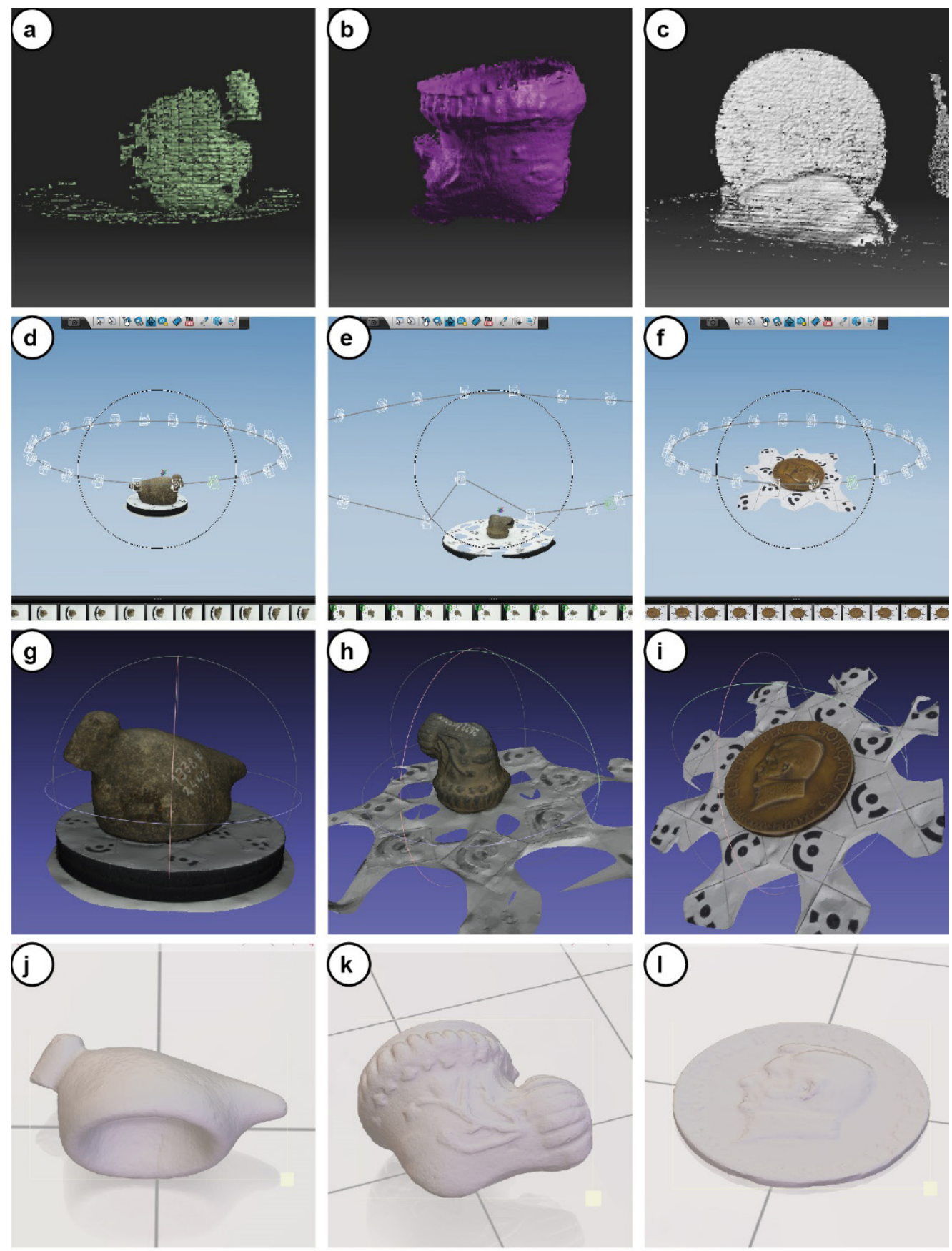

(m)

(n)
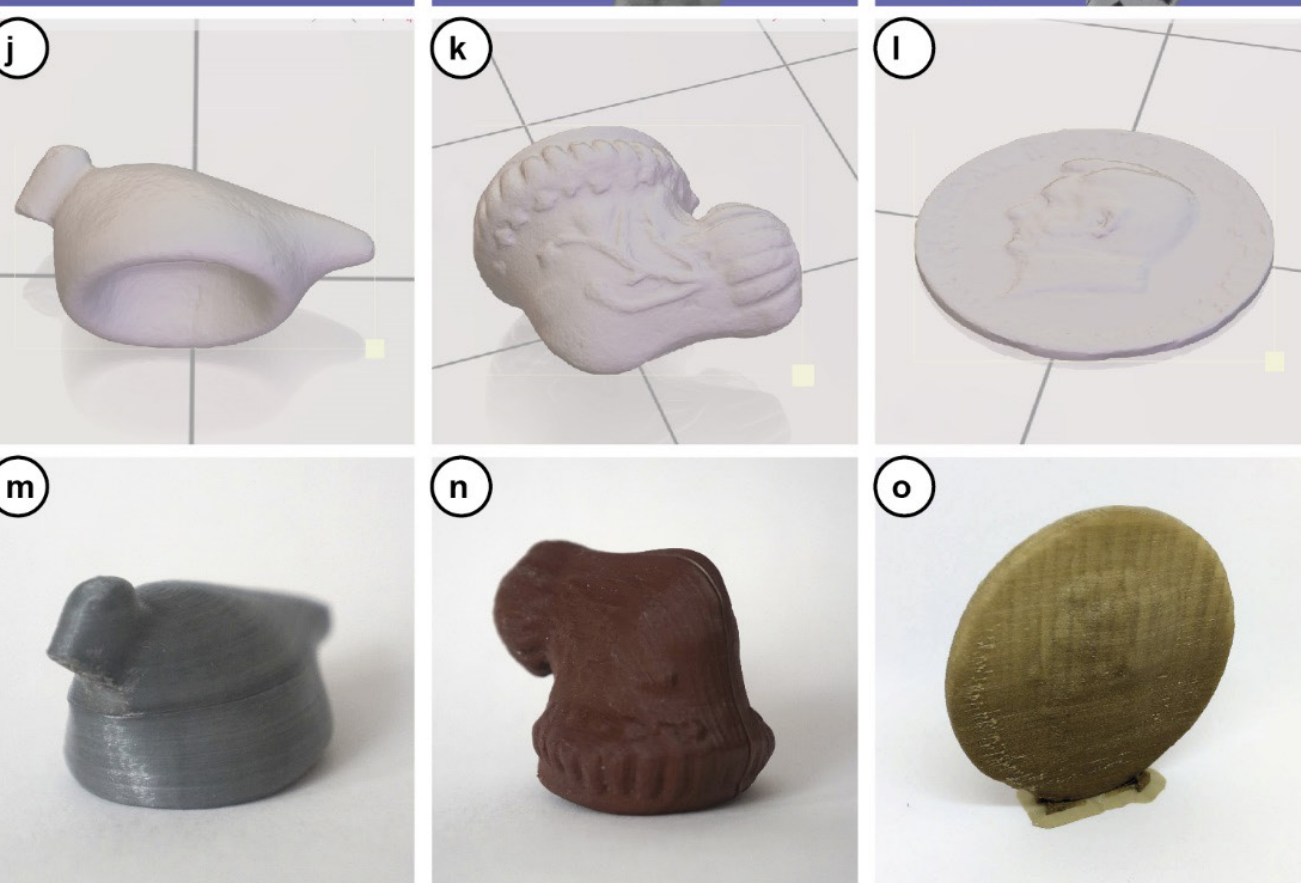

(0)

Zoólito, Cachimbo e medalha: (a, b, c) Processamento no David Laserscanner; (d, e, f) Processamento no 123D Catch; (g, h, i) Tratamento dos modelos fotogramétricos no Meshlab; (j, k, l) Visualização dos modelos fotogramétricos no 3D Builder; $(m, n, 0)$ Modelos fotogramétricos impressos em 3D. Fonte: Elaborada pelos autores. 


\subsection{Fotogrametria com a utilização do 123D Catch}

Foram capturadas sequências de 24 fotos (a cada $15^{\circ}$ ) para cada posição dos objetos. Para o zoólito e o cachimbo, foram realizadas três posições diferentes para cada um. Para a medalha, apenas duas posições foram necessárias, frente e verso.

Após a captura das fotos, realizada durante uma tarde, o programa 123D Catch identificou automaticamente as informações da superfície da medalha e em dois dos ângulos do zoólito (Figura 4d). Já para o cachimbo, foi necessário o realinhamento manual das três posições capturadas (figura 4e). O tempo do processo ficou em torno de doze horas. Ainda durante o processamento, o brilho do metal dificultou a leitura da medalha. O problema foi resolvido com o uso de um filtro polarizador circular (figura 4f).

Durante o pós-processamento, realizado numa tarde, o software livre Meshlab foi utilizado para alinhar as malhas geradas, fazer pequenos reparos e gerar os objetos digitalizados incluindo suas cores (figura 4g, 4h e 4i). Os objetos 3D digitalizados (figuras 4j, 4k, 4I) foram impressos em plástico poliácido láctico (PLA), e o resultado pode ser visto nas figuras $4 \mathrm{~m}$, 4n e 40.

Foi observado que o 123D Catch reduziu a resolução das imagens para $2120 \times 1413$ pixels, uma redução de até $60 \%$ em relação às imagens capturadas, fato que influencia o resultado final para objetos com alto nível de detalhamento.

\section{DISCUSSÃO}

Ao se tratar de técnicas de digitalização 3D faça-você-mesmo, espera-se o baixo custo. 0 custo inicial de implementação de um projeto com qualquer uma das duas técnicas testadas neste trabalho, (contabilizando equipamento fotográfico ou licença de software) varia de $\mathrm{R} \$ 2.000,00$ até $\mathrm{R} \$ 3.500,00$. Porém, tendo em vista o avanço tecnológico que vem tornando acessíveis câmeras digitais de alto desempenho, há museus que já têm o equipamento fotográfico ${ }^{9}$ para o uso na documentação bidimensional, o que resulta numa redução de custos para implementação da fotogrametria.

As técnicas testadas apresentaram uma diferença no tempo de digitalização. Das três etapas processuais da fotogrametria- captura das imagens, processamento, e pós-processamento do modelo obtido- na digitalização tridimensional a laser, no David 3D, o processamento acontece simultaneamente à captura, sem uso de conexão com a internet. Desse modo, o processo de digitalização a laser testado se mostrou mais rápido do que a fotogrametria.

Outro ponto, que também influencia o tempo de digitalização, é a habilidade necessária para utilizar a técnica. Seria leviano afirmar, com base apenas no experimento realizado, qual técnica requer maior habilidade de quem a executa. Entretanto, considerando a sociedade contemporânea, na qual a fotografia é amplamente disseminada (NOTE, 2011), existem uma transferência de aprendizagem ${ }^{10}$ (ELLIS, 1965) para a fotogrametria, o que acaba por reduzir a curva de aprendizagem ${ }^{11}$ nessa técnica 3D (LEARNING CURVE, s.d.). A digitalização a laser, por outro

\footnotetext{
${ }^{9}$ Exemplo: o Museu Júlio de Castilhos tem uma câmera Sony A3000 que tem características adequadas para a fotogrametria.

${ }^{10}$ Conhecimento proveniente de experiências anteriores do usuário que pode ser utilizado para auxiliá-lo num diferente contexto (ELLIS, 1965).

${ }^{11}$ Uma curva de aprendizado é um gráfico que representa a aprendizagem de uma habilidade contra o tempo necessário para aprendê-la (LEARNING CURVE, s.d.).
} 
lado, demanda uma curva aprendizagem maior, apresentando maior dificuldade ao aprendiz. Nestse sentido, inicialmente, a fotogrametria tende a se mostrar mais fácil e mais rápida para obtenção de um modelo digital.

Ao se considerar que o usuário já tem conhecimento de ambas técnicas testadas, a presença de uma etapa a mais no processo pode refletir no tempo de digitalização. Em contrapartida, dentro das etapas da fotogrametria, as imagens adquiridas no processo, paralelamente, constituem uma extensa documentação 2D do patrimônio digitalizado. Além do mais, uma vez que o processamento é uma etapa separada, pode-se realizá-lo conforme viabilidade ou necessidade.

Foi verificado, também, que o tamanho dos objetos experimentados favoreceu a fotogrametria, pois a câmera fotográfica utilizada possui configuração para capturar imagens macro fornecendo dados com a nitidez necessária. Assim, sabendo-se as dimensões do objeto de antemão, é possível fazer uma configuração com lentes adequadas. No caso da digitalização 3D por triangulação a laser, quanto menor o objeto, menor devem ser as marcas de calibração, e mais próximo deve ser posicionada a webcam. Além de a webcam utilizada não ter função macro, a profundidade de campo é reduzida, diminuindo também a amplitude do foco. Tais restrições resultam no aumento da dificuldade em calibrar e do número de varreduras da superfície necessárias.

Em relação ao resultado final, para ambas as técnicas, a qualidade da reconstrução 3D variou entre um objeto e outro. Na fotogrametria, o cachimbo necessitou de alinhamento manual que poderia ter sido evitado na captura de posições em ângulos diferentes. Na digitalização a laser, a instabilidade dos painéis prejudicou a aquisição. Atribuíram-se essas variações à influência de quatro fatores: especificidades da técnica, experiência humana, características materiais e forma dos objetos. Desse modo, verificou-se a necessidade de um novo experimento que compare o nível de influência desses fatores entre as técnicas faça-você-mesmo com as de uso profissional.

A respeito da radiação luminosa, adotada como critério de exclusão de materiais nesse experimento, ambas as técnicas ultrapassam até 100\% a iluminação máxima aconselhada para objetos com sensibilidade à luz. Em contrapartida, foi verificado que as regras de conservação preventiva são relacionadas ao ambiente de armazenagem da peça e, sendo assim, a incidência e intensidade luminosa a longo prazo. Nesse sentido, vale a pena considerar a importância da obtenção do objeto 3D digitalizado tendo em vista o curto tempo de exposição luminosa, pois são necessários alguns minutos durante a captura de dados, seja na fotogrametria ou na digitalização a laser.

Observa-se, ainda, que o software de processamento fotogramétrico utilizados nos ensaios, Autodesk 123D Catch, foi descontinuado pela desenvolvedora em abril de 2017. Funções semelhantes, além de ferramentas de processamento adicionais, podem ser encontradas no software Autodesk Recap, disponível em versão gratuita ou comercial.

\section{CONCLUSÃO}

Tanto a fotogrametria quanto a digitalização 3D por triangulação a laser faça-você-mesmo mostraram ter condições de implementação para o uso na preservação do patrimônio cultural. Porém ambas apresentaram, durante o experimento, aspectos que precisam ser melhorados.

A digitalização 3D por triangulação a laser faça-você mesmo mostrou, como desvantagem, o processo de calibração (fator de especificidades da técnica e experiência humana). Os painéis precisam de um material que promova estabilidade e resistência no decorrer dos usos para a 
obtenção de uma calibragem de boa qualidade a longo prazo. Outra desvantagem é relacionada à preocupação dos profissionais em relação à conservação devido ao laser ser comumente associado a ferramentas invasivas (fator de experiência humana). Sua vantagem está no tempo de digitalização reduzido, mas apenas se executada por um usuário experiente.

A fotogrametria faça-você-mesmo mostrou, como ponto negativo, a configuração para a captura das imagens influenciável pela perícia do usuário (fator de experiência humana). Sua principal vantagem está na necessidade de uma menor curva de aprendizagem e no compartilhamento de tarefas com a documentação 2D facilitando seu uso por profissionais não especialistas na técnica.

Em paralelo, requisitos do 123D Catch e do David Laserscanner 3D que reduzem a resolução do modelo digitalizado sugerem a necessidade de testar outros softwares (fator de especificidades da técnica).

Ambas as técnicas testadas têm dificuldades para digitalizar objetos de materiais com alta refletância ou transparentes (fator características materiais do objeto). No estado atual da arte, as possíveis soluções para contornar esse problema implicam aumento de custo e de especialização do usuário.

Após observar os fatores apontados, a fotogrametria mostrou-se a melhor alternativa para a implementação da digitalização 3D faça-você-mesmo em museus. Após os técnicos do museu se habituarem com a técnica, uma avaliação do acervo deve ser feita, e a configuração para fotogrametria pode ser aprimorada. Esse aprimoramento pode envolver a incorporação de câmeras e fontes luminosas adicionais, assim como plataformas giratórias motorizadas e outros recursos para a automação do processo.

O uso da digitalização 3D faça-você-mesmo nos museus apresentou-se como oportunidade para a implementação da preservação digital do patrimônio cultural de forma acessível. Isso possibilita ao técnico da instituição se apropriar dos conhecimentos dos métodos e criar um ambiente propício para desenvolver uma aplicação tendo em vista a inovação tecnológica dos processos museológicos. O objeto preservado digitalmente favorece também a educação patrimonial e mesmo a difusão cultural, possibilitando novas formas de acesso ao conhecimento sem barreiras físicas, geográficas, ou de tempo em meio digital. Assim, propicia-se ao público uma nova visão do acervo, e ao museu, um acervo mais reconhecido.

\section{REFERÊNCIAS}

ANDRADE, B. T. et al. 3D preserving XVIII century barroque masterpiece: challenges and results on the digital preservation of Aleijadinho's sculpture of the prophet joel. Journal of Cultural Heritage, v. 13, n. 2, p. 210-4, abr. 2012.

AZEVEDO, S. A. K. et al. Processos de Digitalizacao 3D e prototipagem física utilizados no Laboratório de processamento de Imagem digital do Museu Nacional/ UFRJ. In: CONGRESSO LATINO AMERICANO PALEONTOLOGIA DE VERTEBRADOS, 4., 2011, San Juan, Argentina. Anais [...]. San Juan, Argentina, 2011.

BERNARDINI, F.; RUSHMEIER, H. The 3D Model Acquisition Pipeline. Computer Graphics Forum, v. 21, p. 149-72, 2002.

BOSCHETTO, A.; BOTTINI, L.; VENIALI, F. Integration of FDM surface quality modeling with process design. Additive Manufacturing, v. 12, parte B, p. 334-44, out. 2016. 
BRASIL. Ministério do Desenvolvimento, Indústria e Comércio. Instituto Nacional de Metrologia, Normalização e Qualidade Industrial INMETRO. Portaria INMETRO n. 064, de 21 de março de 2006. Diário Oficial da União, Brasília. Seção 1, p. 77, 15 abr. 2006.

DANTAS, P. V. de F. et al. Protótipo de dispositivo facilitador para digitalização 3D por fotogrametria com smartphones. In: CONGRESO DE LA SOCIEDAD IBEROAMERICANA DE GRÁFICA DIGITAL, 20., 2016, Buenos Aires. Blucher Design Proceedings. São Paulo: Blucher, 2016. v. 3, p. 297-304.

EBRAHIM, M. A. 3D Laser Scanners: history, applications, and future. Civil Engineering Department. Faculty of Engineering. Assiut: Assiut University, 2011.

ELLIS, H. C. The transfer of learning. New York: The Macmillan Company, 1965. 200p.

GOMES, L. Reconstrução 3D de acervos culturais usando câmeras RGB-D: solução de compromisso entre precisão e tempo aplicada ao projeto Aleijadinho digital. 2016. Tese (Doutorado em Informática) - Universidade Federal do Paraná (UFPR), Curitiba, 2016.

GUARNIERI, A.; PIROTTI, F.; VETTORE, A. Cultural heritage interactive 3D models on the web: an approach using open source and free software. Journal of Cultural Heritage, v. 11, n. 3, p. 350-3, jul./set. 2010.

LEARNING CURVE. In: Business Dictionary, s.d. Disponível em: http://www.businessdictionary.com/ definition/learning-curve.html. Acesso em: 12 fev. 2018.

LEMOS, A. Cibercultura. 2. ed. Porto Alegre, RS: Sulina, 2004.

LEVOY, M. et al. The Digital Michelangelo Project: 3D Scanning of Large Statues. In: SIGGRAPH. Proceedings of the 27th annual Conference on Computer Graphics and Interative Techniques, 2000. p. 1-14. Disponivel em: https://graphics.stanford.edu/papers/dmich-sig00/dmich-sig00-nogamma-comp-low.pdf. Acesso em: 20 abr. 2017.

MUCHACHO, R. M. S. P. Museu e novos media: a redefinição do espaço museológico. 2009. 114 f. Dissertação (Mestrado em Museologia) - Universidade Lusófona de Humanidades e Tecnologias, Lisboa, 2009. Disponível em: http://www.museologia-portugal.net/files/upload/mestrados/rute_muchacho.pdf. Acesso em: 15 maio 2016.

NOTE, M. Photographic image history. In: NOTE, M. Managing image collections: a practical guide. Oxford: Chandos Publishing, 2011. Chandos Information professional series, p. 15-37.

PAVLIDIS, G. et al. Methods for 3D digitization of Cultural Heritage. Journal of Cultural Heritage, v. 8, n. 1, trimestral, p. 93-9, 2007.

PERCOCO, G.; LAVECCHIA, F.; SALMERÓN, A. J. S. Preliminary study on the 3D digitization of millimeter scale products by means of photogrammetry. Procedia CIRP, v. 33, p. 257-62, 2015.

REFLETÂNCIA. In: Dicionário Priberam da Língua Portuguesa, [s.d.]. Disponível em: https://www.priberam. pt/dlpo/reflect\%C3\%A2ncia. Acesso em: 15 ago. 2017.

REPRAP. 3D scanning. 2016. Disponível em: http://reprap.org/wiki/3D_scanning. Acesso em: 25 ago. 2016.

SCHENK, T. Introduction to photogrammetry. Columbus: The Ohio State Universitiy, 2005. 95p. Disponível em: http://www.mat.uc.pt/ gil/downloads/IntroPhoto.pdf. Acesso em: 28 nov. 2016.

SILVA, F. P. da. Usinagem de espumas de poliuretano e digitalização tridimensional para fabricação de assentos personalizados para pessoas com deficiência. 2011. 196 f. Tese (Doutorado em Engenharia) - 
Universidade Federal do Rio Grande Sul (UFRGS), Porto Alegre, 2011. Disponível em: http://www.lume. ufrgs.br/handle/10183/36040. Acesso em: 8 maio 2016.

SILVA, F. P.; KINDLEIN JUNIOR, W. O Laçador = The Lassoer. In: SANTOS, J. R. L. et al. (Org.). Tecnologias 3D: desvendando o passado, modelando o futuro $=3 \mathrm{D}$ Technologies: unveiling the past, shaping the future. 1. ed. Rio de Janeiro: Lexicon, 2013. v. 1, p. 210-5.

SOUZA, L. A. C.; FRONER, Y. Reconhecimento de materiais que compõem acervos. 4. ed. Belo Horizonte: Lacicor, 2008. 31p.

TOMMASELLI, A. M. G. et al. Fotogrametria: aplicações a curta distância. In: MENEGUETTI JR., M.; ALVES, N. (Org.). FCT 40 anos: perfil científico-educacional. Presidente Prudente, SP: UNESP, 1999. p. 147-59.

WEINMANN, M.; KLEIN, R. Advances in geometry and reflectance acquisition (course notes). In: SIGGRAPH. Asia 2015 Courses. New York: ACM, 2015. 71p. Disponível em: http://doi.acm. org/10.1145/2818143.2818165. Acesso em: 19 out. 2016.

YASTIKLI, N. Documentation of cultural heritage using digital photogrammetry and laser scanning. Journal of Cultural Heritage, v. 8, n. 4, p. 423-7, 2007.

\section{Sobre autores:}

Yvana Oliveira Alencastro: Mestra em Design e Tecnologia no Programa de pós-graduação em Design da Universidade Federal do Rio Grande do Sul (UFRGS). MBA em Marketing realizado na Universidade de Pernambuco (UPE). Especialista em Design de Interação para artefatos digitais realizado no Centro de Estudos e Sistemas Avançados do Recife (CESAR). Graduada em Design pela Universidade Federal de Pernambuco (UFPE). Designer do Serviço Nacional de Aprendizagem Industrial - Instituto SENAI de lovação para Tecnologias da Informação e Comunicação. Membro do grupo de pesquisa Núcleo de Desenvolvimento de Produtos (NDP). E-mail: yvana.alencastro@ gmail.com, Orcid: http://orcid.org/0000-0002-0984-2920

Paulo Victor de Farias Dantas: Mestre em Design e Tecnologia no Programa de pós-graduação em Design da Universidade Federal do Rio Grande do Sul (UFRGS), vinculado ao Laboratório de Design e Seleção de Materiais (LdSM), desenvolvendo pesquisas nas linhas de "Digitalização Tridimensional" e "Design e Patrimônio Cultural", e colabora com o Laboratório de Pesquisas em Fotogrametria (LAFOTO-UFRGS). Graduado em Escultura pela Escola de Belas Artes da Universidade Federal do Rio de Janeiro (EBA/UFRJ). Graduado no curso tecnológico Design Gráfico: Ilustração e Animação Digital, na Universidade Veiga de Almeida (UVA). E-mail: victor.dantas@ufrgs.br, Orcid: http://orcid.org/0000-0002-0231-2599

Fábio Pinto da Silva: Doutor e mestre em Engenharia, com ênfase em Ciência e Tecnologia de Materiais, pelo PPGE3M da Universidade Federal do Rio Grande do Sul (UFRGS). Graduado em Engenharia Mecânica pela UFRGS. Professor adjunto no Departamento de Design e Expressão Gráfica (DEG/FA/UFRGS) e professor permanente no Programa de Pós-Graduação em Design, com ênfase em Design \& Tecnologia (PGDESIGN/UFRGS). Consultor do CNPq, CAPES e FAPERGS. Coordenador do Centro Multiusuário de Prototipagem Rápida (CMPR), do Parque Científico e Tecnológico da UFRGS (ZENIT/UFRGS). Membro do comitê gestor do Laboratório de Inovação e Fabricação Digital da Escola de Engenharia (LIFEE/UFRGS). Integrante do Comitê Local de Iniciação Tecnológica, da Secretaria de Desenvolvimento Tecnológico (SEDETEC/UFRGS). 
Pesquisador no Laboratório de Design e Seleção de Materiais (LDSM/UFRGS). E-mail: E-mail: fabio.silva@ufrgs.br, Orcid: http://orcid.org/0000-0001-9349-5601

Jocelise Jacques de Jacques: Doutora em Engenharia de Produção pela Universidade Federal do Rio Grande do Sul (UFRGS), tendo desenvolvido parte de sua pesquisa na Universidade da Califórnia - Berkeley. Mestre em Engenharia Civil e graduação em Arquitetura e Urbanismo pela UFRGS. Professora adjunta da UFRGS, na Faculdade de Arquitetura, Departamento de Design e Expressão Gráfica. Participa da Comissão de Graduação em Design de Produto. Professora colaboradora do Programa de Pós-Graduação em Design (UFRGS).E-mail: jocelise.jacques@ufrgs.br, Orcid: http://orcid.org/0000-0003-2109-0677 